\title{
A patient-level meta-analysis of studies evaluating vagus nerve stimulation therapy for treatment-resistant depression
}

This article was published in the following Dove Press journal:

Medical Devices: Evidence and Research

28 February 2013

Number of times this article has been viewed

\section{Scott M Berry' \\ Kristine Broglio' \\ Mark Bunker ${ }^{2}$ \\ Amara Jayewardene 2 \\ Bryan Olin²}

A John Rush ${ }^{3}$

'Berry Consultants, Austin, TX, USA; ${ }^{2}$ Cyberonics, Inc, Houston, TX, USA; ${ }^{3}$ Duke-NUS, Office of Clinical Sciences, Singapore
Correspondence: Bryan Olin

Clinical, Quality and Regulatory,

Cyberonics, Inc, 100 Cyberonics Blvd,

Houston, TX 77058, USA

Tel +I 28I 2287323

Fax + I 28I 8532560

Email bryan.olin@cyberonics.com
Objective: To compare response and remission rates in depressed patients with chronic treatment-resistant depression (TRD) treated with vagus nerve stimulation (VNS) Therapy ${ }^{\mathbb{B}}$ plus treatment as usual (VNS + TAU) or TAU alone in a meta-analysis using Bayesian hierarchical models.

Data sources and study selection: Six outpatient, multicenter, clinical trials that have evaluated VNS + TAU or TAU in TRD, including two single-arm studies of VNS + TAU $(n=60$ and $n=74)$, a randomized study of VNS + TAU versus TAU $(n=235)$, a randomized study of VNS + TAU comparing different VNS stimulation intensities $(n=331)$, a nonrandomized registry of VNS + TAU versus TAU $(n=636)$, and a single-arm study of TAU $(n=124)$ to provide longer-term, control data for comparison with VNS-treated patients.

Data extraction: A systematic review of individual patient-level data based on the intent-to-treat principle, including all patients who contributed more than one post-baseline visit. Response was based on the Montgomery-Åsberg Depression Rating Scale (MADRS) and the Clinical Global Impressions scale's Improvement subscale (CGI-I), as these were the two clinician-rated measures common across all or most studies. Remission was based on the MADRS.

Results: Outcomes were compared from baseline up to 96 weeks of treatment with VNS + TAU $(n=1035)$ versus TAU $(n=425)$. The MADRS response rate for VNS + TAU at $12,24,48$, and 96 weeks were $12 \%, 18 \%, 28 \%$, and $32 \%$ versus $4 \%, 7 \%, 12 \%$, and $14 \%$ for TAU. The MADRS remission rate for VNS + TAU at 12, 24, 48, and 96 weeks were 3\%, 5\%, $10 \%$, and $14 \%$ versus $1 \%, 1 \%, 2 \%$, and $4 \%$, for TAU. Adjunctive VNS Therapy was associated with a greater likelihood of response (odds ratio $[\mathrm{OR}]=3.19,95 \%$ confidence interval $[\mathrm{CI}]: 2.12,4.66)$ and remission $(\mathrm{OR}=4.99, \mathrm{CI}: 2.93,7.76)$, compared with TAU. For patients who had responded to VNS + TAU at 24 weeks, sustained response was more likely at 48 weeks $(\mathrm{OR}=1.98, \mathrm{CI}: 1.34,3.01)$ and at 96 weeks $(\mathrm{OR}=3.42$, CI: 1.78, 7.31). Similar results were observed for CGI-I response.

Conclusion: For patients with chronic TRD, VNS + TAU has greater response and remission rates that are more likely to persist than TAU.

Keywords: Bayesian meta-analysis, remission rate, response rate, treatment-resistant depression, vagus nerve stimulation, VNS Therapy

\section{Introduction}

Depression is a prevalent, disabling, and often chronic or recurrent psychiatric condition affecting about 350 million people worldwide. ${ }^{1}$ Among the general adult population in the US, the lifetime prevalence of major depressive disorder (MDD) is about $29.9 \%$ and the 12 -month prevalence is about $8.6 \% .^{2}$ MDD imposes significant costs on patients, their families, caregivers, employers, and insurance payers, with an estimated 
direct cost of more than US\$80 billion per year to the US economy, which includes costs associated with health care, suicide mortality, and lost workplace productivity. ${ }^{3,4}$

While several modalities have shown effectiveness in the treatment of a major depressive episode, a recent, large-scale prospective trial evaluating over 3000 subjects demonstrated that almost $35 \%$ of outpatients with nonpsychotic MDD do not respond to four or more conventional treatment approaches, and are therefore considered to have treatment-resistant depression (TRD). ${ }^{5}$

VNS Therapy ${ }^{\circledR}$ (Cyberonics, Inc, Houston, TX, USA) consists of a small pulse generator surgically implanted in the chest that delivers intermittent electrical signals via an electrode partially wrapped around the left vagus nerve in the mid-cervical region; the electrical signals are in turn processed in the nucleus tractus solitarius and relayed to various regions of the brain. ${ }^{6}$ In 1997, the US Food and Drug Administration (FDA) approved VNS Therapy for the adjunctive treatment of drug-resistant epilepsy, and, in 2005, it was approved for the adjunctive long-term treatment of chronic or recurrent depression for adult patients experiencing a major depressive episode who had failed to respond to four or more adequate antidepressant treatments.

The positive health economic benefits of VNS Therapy for drug-resistant epilepsy have been well documented in the literature. ${ }^{7-11}$ Cohen and Allen developed a payerperspective formula to estimate the potential reductions in health care utilization costs with adjunctive VNS Therapy in TRD. ${ }^{12}$ More recently, Feldman et al conducted an analysis of the Medicare claims database, which showed positive health outcomes and cost savings for Medicare beneficiaries with TRD who are treated with adjunctive VNS Therapy. ${ }^{13}$

A limited amount of postmarketing data is available for continued assessment of the effectiveness of VNS Therapy in treating patients with TRD. Two recent publications systematically reviewed the available literature, ${ }^{14,15}$ but the publications were limited as they did not address patient-level data and also did not include data from Study D-21 (an acute [22-week] and long-term [50-week] study including 331 patients with TRD receiving open-label VNS Therapy; the data has subsequently been published); ${ }^{16}$ Study D-23 (a long-term [60-month], open-label, observational registry including data on 335 patients receiving VNS Therapy plus treatment as usual [VNS + TAU] and 301 patients receiving TAU [Cyberonics Inc, data on file, 2011]); and longer-term data from older studies of VNS Therapy that have not been published (Cyberonics Inc, data on file, 2005).
However, both the aforementioned publications identified the need for an additional study analyzing the effect of VNS in TRD. ${ }^{14,15}$

To address this call for additional data, we conducted a meta-analysis that systematically evaluated patient-level data from six prospective, outpatient, multicenter, clinical studies evaluating treatment with either adjunctive VNS + TAU or TAU alone in a TRD patient population. In these studies, TAU included any concomitant treatment strategy that the patients and their psychiatrists chose to follow, including psychotropic medications and nonpharmacologic treatments (such as psychotherapy, cognitive behavioral therapy, bright light therapy, and electroconvulsive therapy [ECT]); additionally, the concomitant treatment strategies could be adjusted, started, and stopped, as necessary.

Briefly, the six clinical studies included in the meta-analysis were two single-arm studies of VNS + TAU (Studies D-01 and D-03), a randomized trial of VNS + TAU versus TAU (Study D-02), a single arm study of patients who received TAU (Study D-04), a randomized trial of VNS + TAU comparing different VNS stimulation intensities (Study D-21), and a nonrandomized registry of patients who received either VNS + TAU or TAU (Study D-23).

To analyze the efficacy of VNS Therapy, raw scores and response and remission rates for the two treatments - VNS + TAU versus TAU - were modeled using a Bayesian hierarchical model for repeated measures data. Response was assessed across all studies using the 10-item Montgomery-Åsberg Depression Rating Scale (MADRS) and the Clinical Global Impressions scale's Improvement subscale (CGI-I). ${ }^{17,18}$ Remission was assessed using the MADRS.

\section{Methods}

\section{Patients}

All enrolled patients had nonpsychotic MDD (recurrent or single episode) or depressed phase, bipolar I or II disorders, and were experiencing a nonpsychotic, major depressive episode at the time of study enrollment. Per study protocol and guidelines from the institutional ethics committees, all patients provided written informed consent prior to enrollment. Per the investigator's judgment, patients undergoing VNS implantation had to be generally healthy for the surgical procedure (including anesthesia requirements) and a VNS Therapy System implant could not be considered to pose an unacceptable surgical or medical risk for the patient. Patient eligibility criteria for each study are summarized in Table S1. 


\section{Description of the clinical studies included in the meta-analysis}

Six prospective, outpatient, multicenter, clinical studies with patient-level data were included in the meta-analysis; no further selection criteria were necessary as these essentially represent the totality of studies of VNS Therapy in this patient population. The studies were sponsored by Cyberonics, Inc, to evaluate VNS Therapy in a TRD patient population. Efficacy was evaluated in these studies using various assessment tools, including the MADRS and CGI-I. Specifically, the MADRS and CGI-I were secondary outcome measures in Studies D-01, D-02, D-03, D-21, and D-23; and the CGI-I (but not MADRS) was a secondary outcome measure in Study D-04. Although the MADRS and CGI-I were secondary outcome measures in these studies, they have been utilized as the primary outcomes for the meta-analysis as these were the only clinician-rated assessment tools common to the majority of the studies; that is, five of the six studies used the MADRS and all six studies used the CGI-I (Table S2).

Detailed descriptions and the outcomes of Studies D-01, D-02, D-03, D-04, and D-21 have been previously published as cited in the following descriptions of the studies, but results from the TRD Registry (D-23) are not currently published. Only Studies D-02 and D-21 were randomized trials, and note that only TAU studies that were conducted for comparison to VNS treatment were included in the meta-analysis to ensure similarity of patient populations. All studies were conducted at multiple centers in the US and Canada, except for Study D-03, which was conducted at multiple centers in Europe. Following are synopses of the results from the various studies:

- D-01 was an open-label, observational, and longitudinal study evaluating VNS + TAU treatment over 24 months. ${ }^{19-21}$ Data from 60 patients receiving VNS + TAU were included in the meta-analysis.

- D-02 was a pivotal, randomized, sham-controlled study evaluating VNS in patients with TRD. ${ }^{22,23}$ Prior to the start of the acute 12-week phase of the study, 235 patients were implanted with the VNS Therapy device: of these, 119 patients were randomly allocated to have the device activated at a specified dose and 116 patients were randomly allocated to the sham control arm in which the implanted device was not activated. After the acute phase was completed, the device was activated for these 116 patients. The acute phase included an initial 2-weeklong period following implant when the VNS device was not turned on to aid recovery. The 12-week acute phase was followed by a 9-month open-label treatment phase when VNS devices were activated for the patients who were previously in the sham group. Data from 235 patients receiving VNS + TAU were included in the meta-analysis.

- D-03 was a 2-year, observational, open-label, longitudinal study evaluating VNS + TAU. ${ }^{24,25}$ Data from 74 patients receiving VNS + TAU were included in the meta-analysis.

- D-04 was an observational, open-label, longitudinal study of TAU. ${ }^{26,27}$ For 2 years, the study tracked the outcomes of 124 patients who received TAU (included in the meta-analysis). The majority of these patients were recruited from the same centers as those participating in the D-02 study. They were chosen to provide a longer-term control group of similarly ill subjects who received the usual standard-of-care therapies but were not implanted with the VNS Therapy device for comparison to the VNS + TAU patients in the D-02 study.

- D-21 was a post-approval study that evaluated different doses of VNS Therapy. ${ }^{16}$ During the acute phase (22-weeks long), 331 patients were randomized to one of three dosing groups represented by the amount of electrical charge delivered to the vagus nerve: low dose $(0.25 \mathrm{~mA}, 130 \mu \mathrm{sec})$, medium dose $(0.5-1.0 \mathrm{~mA}$, $250 \mu \mathrm{sec})$, and high dose (1.25-1.5 mA, $250 \mu \mathrm{sec})$. The 50 -week long-term phase was designed as an observational, open-label, longitudinal, multicenter study of VNS + TAU and included 298 completed patients. ${ }^{16}$ Data from 331 patients receiving VNS + TAU were included in the meta-analysis.

- D-23 is an ongoing, long-term (60-month), open-label, observational registry of patients receiving VNS + TAU or TAU (ClinicalTrials.gov Identifier: NCT00320372; Cyberonics, Inc, data on file, 2011). Data from 335 patients receiving VNS + TAU and 301 patients receiving TAU were included in the meta-analysis. The clinicians assessed the patients' conditions regarding the severity of illness using the CGI-I and MADRS.

\section{Patients and ethics}

The respective institutional review boards (IRB) or ethics committees (EC) approved the studies. Written informed consent was obtained from all enrolled patients using IRB/ EC-approved informed consent forms.

\section{Efficacy assessment}

Multiple depression rating scales were used in each study to measure depression symptoms. Depression symptoms were 
typically assessed at baseline and at weeks 12, 24, 48, 72, and 96; however, as mentioned in the "Statistical analyses" section, actual visit dates were used in the repeated measures analysis. The MADRS and CGI-I were selected as the primary endpoints for the meta-analysis as these are commonly used and validated scales for measuring depression symptomatology, and CGI-I was assessed in all six studies and MADRS in five of the studies.

Outcomes of interest over time were response, remission, and sustained response based on the MADRS and CGI-I and the following definitions:

- Response using the MADRS: a patient was considered to be a responder if their MADRS score at the follow-up visit was at least a $50 \%$ reduction from their baseline MADRS score.

- Response using the CGI-I: a patient was considered to be a responder if their CGI-I score at the follow-up was 1 ("very much improved") or 2 ("much improved").

- Remission using the MADRS: a patient was considered to be in remission if their MADRS score at the follow-up was $<10$ points.

\section{Safety assessment}

Safety was evaluated based on adverse events (AEs) reported for all patients following implantation and at each scheduled follow-up study visit during participation in Studies D-01, D-02, D-03, and D-21. Note that data up to 12 months are available from Study D-21 and no AE reports were collected in Studies D-04 (evaluating a TAU group) and D-23 (evaluating TAU versus VNS + TAU groups).

\section{Statistical analyses}

This meta-analysis is based on individual patient-level data and includes all existing multicenter studies of VNS + TAU or TAU in TRD patient populations. Because only one of the studies randomized patients to VNS + TAU or TAU (ie, the acute phase of the D-02 study), we used propensity scores to control for any potential differences between the treatment groups.

Propensity scores were calculated by fitting a logistic regression model using SAS PROC GLIMMIX with the dependent variable of treatment group $(1=\mathrm{VNS}+\mathrm{TAU}$ and $0=\mathrm{TAU}$ ). The regression model included the following measured baseline variables: age, gender, ethnicity, duration from first diagnosis of illness, duration since diagnosis of current episode, number of prior medications by Anatomical Therapeutic Chemical classifications, ${ }^{28}$ ECT history (yes or no), baseline MADRS and CGI's Severity subscale (CGI-S) scores, number of previous psychiatric hospitalizations, and number of lifetime suicide attempts. The propensity score is the fitted probability of the response (VNS + TAU group) from the multivariate regression model for each observation (patient) in the data set. The fitted probabilities are ranked into five categories (quintiles) using SAS PROC RANK (SAS Institute Inc, Cary, NC, USA).

To compare VNS + TAU versus TAU, we fitted mixed effects repeated measures models to account for the staggered visit schedules. The fixed terms included an intercept (or grand mean), treatment effect, and propensity score; no treatment-by-time interaction was considered due to difficulties with interpretation and identifiability issues posed by the large number of time points. We took a Bayesian approach that modeled both studies and subjects as random effects by placing a hierarchical model on these terms. ${ }^{29}$ Week of follow-up was modeled with a normal dynamic linear model (NDLM). ${ }^{30}$ The NDLM accounts for the correlation between time points and borrows information across observations such that, for observations closer together in time, more information is borrowed, and for observations further apart in time, less information is borrowed. Noninformative priors were assumed for all parameters. For MADRS and CGI-I scores, we modeled the mean score for subjects as a function of the time point and treatment groups. For response and remission endpoints (either MADRS or CGI-I), we modeled the log-odds and report the odds ratios associated with the treatment groups.

Treatment group was considered as categorical with two categories, VNS + TAU and TAU alone. Propensity score was included as a categorical variable. Models were fit including all subjects and a factor for each propensity score and within each subgroup defined by propensity score.

Finally, we compared the proportion of patients who were sustained responders between the two treatment groups. In these analyses, patients were considered sustained responders at 48 weeks if they achieved a response (ie, a 50\% reduction in MADRS score) at both 24 and 48 weeks. Similarly, patients were considered sustained responders at 96 weeks if they achieved a response at both 24 and 96 weeks. Because studies had follow-up visits at slightly different times, visits within 2 weeks of each other were combined for the purposes of this analysis. Sustained response was considered for both the MADRS and CGI-I. We present the raw proportion of patients who were sustained responders and the odds ratio for sustained response of VNS + TAU versus TAU along with the corresponding 95\% confidence interval. 
To aid in assessing clinical benefit, we calculated the numbers needed to treat (NNT) for VNS + TAU based on a comparison of the observed response rates for VNS + TAU and TAU.

Incidence for each $\mathrm{AE}$ preferred term was computed as a proportion of subjects that experienced the event within each year. A subject who experienced the same event more than once within year 1 or year 2 was counted once for each preferred term within each year. An AE that occurred in the first year of VNS treatment, but did not resolve until the second year, was counted as occurring in both the first and second years of treatment.

Analyses were conducted with a self-written code using Intel Fortran compiler v11.0.083 (The Fortran Company, Santa Clara, CA, USA) and using R (The R Foundation for Statistical Computing, Vienna, Austria). Repeated measures models were fit using standard Markov chain Monte Carlo Metropolis-Hastings steps.

\section{Results}

\section{Characteristics of the patient populations}

Intent-to-treat classifications were used in the studies with randomization, and all observations within 2 years, from all patients who were enrolled in one of the six clinical studies.

Table 1 summarizes the baseline characteristics of the study patients by treatment group. These studies were performed over the span of several years, during which time the designs evolved to determine the most important characteristics to assess at baseline. Hence, not every baseline clinical characteristic was collected in every one of the six studies as indicated in Table 1 (eg, number of hospitalizations and number of lifetime suicide attempts were not collected in Study D-01, and the MADRS was not assessed in Study D-04). A total of 1035 patients were treated with VNS + TAU and 541 patients with TAU (the TAU group includes 425 from D-04 and D-23 plus 116 from D-02). Note that the totals for both groups include the 116 patients from the D-02 study: these patients were randomized to the sham-control TAU group for the initial 12-week acute phase (while the VNS devices remained inactive), and, thereafter, the patients' VNS devices were activated and their subsequent long-term phase data were included in the VNS + TAU group, with the week 12 MADRS and CGI-I data considered as baseline for VNS + TAU.

Patients in the two treatment groups were similar in age $(47.8 \pm 10.2$ years for the VNS + TAU group and $48.7 \pm 10.9$ years for the TAU group) and gender distribution $(66.2 \%$ female in VNS + TAU group and $69.7 \%$ female in
TAU group) (Table 1). More non-Caucasian patients were found in the TAU group (9.4\%) than in the VNS + TAU group (3.5\%). The VNS + TAU population had evidence of greater chronicity and treatment resistance; specifically, more patients who received VNS + TAU had ECT (56.1\% versus $39.5 \%$ in the TAU group), more had unsuccessful prior drug treatment trials $(6.9 \pm 2.2$ versus $5.9 \pm 2.2$ in the TAU group), and more had lifetime depression-related hospitalizations ( $3.4 \pm 6.0$ versus $1.9 \pm 4.3$ in the TAU group).

The withdrawal rates during the acute and long-term phases were comparable (23.6\% for VNS + TAU and $22.2 \%$ for TAU) (data not shown). The most common reasons for withdrawal were: patient withdrew consent $(3.4 \%$ for VNS + TAU and $6.3 \%$ for TAU), protocol non-compliance by patient ( $2.1 \%$ for VNS + TAU and $4.9 \%$ for TAU), and lack of efficacy ( $2.3 \%$ for VNS + TAU and $0 \%$ for TAU).

The number of patients per propensity score quintile and their demographic characteristics are presented in Table S3. Basic demographic characteristics were similar. Note the marked gradation of disease state severity from propensity score quintile 1 to quintile 5 . Significant increases across quintiles can be seen from number of hospitalizations, suicidality (prior attempts during lifetime and current episode, as well as, the percentage of suicidal patients at baseline), and baseline assessments (CGI-S and MADRS). Note also that the percentage of patients with a bipolar diagnosis increases with the propensity score quintile.

\section{Repeated measures assessment}

Figure 1 shows the model estimated mean MADRS and CGI-I scores, as well as probabilities of response across follow-up for both treatments. The models did not include a treatment-by-time interaction; thus, these plots show parallel trends over time for both treatment groups. The offset illustrates the better outcomes for patients who received VNS + TAU, while outcomes for both groups tended to improve. Specifically, the model-based MADRS response rates for VNS + TAU at 12, 24, 48, and 96 weeks were $12 \%$, $18 \%, 28 \%$, and $32 \%$ versus $4 \%, 7 \%, 12 \%$, and $14 \%$ for TAU, respectively. Similarly, CGI-I response rates for VNS + TAU at $12,24,48$, and 96 weeks were $14 \%, 23 \%, 40 \%$, and $50 \%$ versus $3 \%, 4 \%, 10 \%$, and $14 \%$ for TAU, respectively. Finally, the MADRS remission rates for VNS + TAU at 12, 24,48 , and 96 weeks were approximately $3 \%, 5 \%, 10 \%$, and $14 \%$ versus $1 \%, 1 \%, 2 \%$, and $4 \%$ for TAU, respectively.

Figures S1 and S2 show MADRS and CGI-I scores by study and treatment group at selected time points. Because follow-up visit schedules varied by study, some visit times 


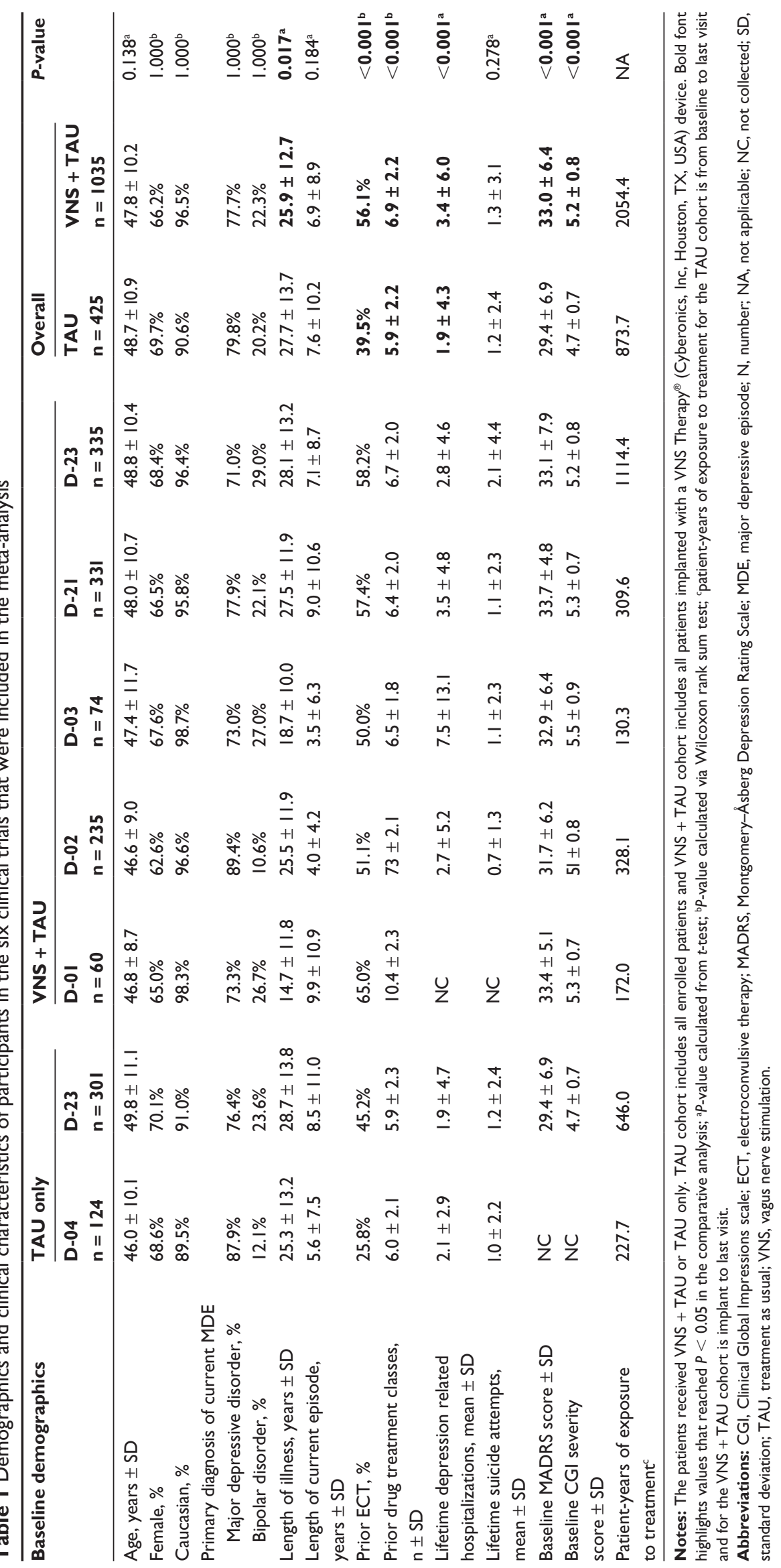



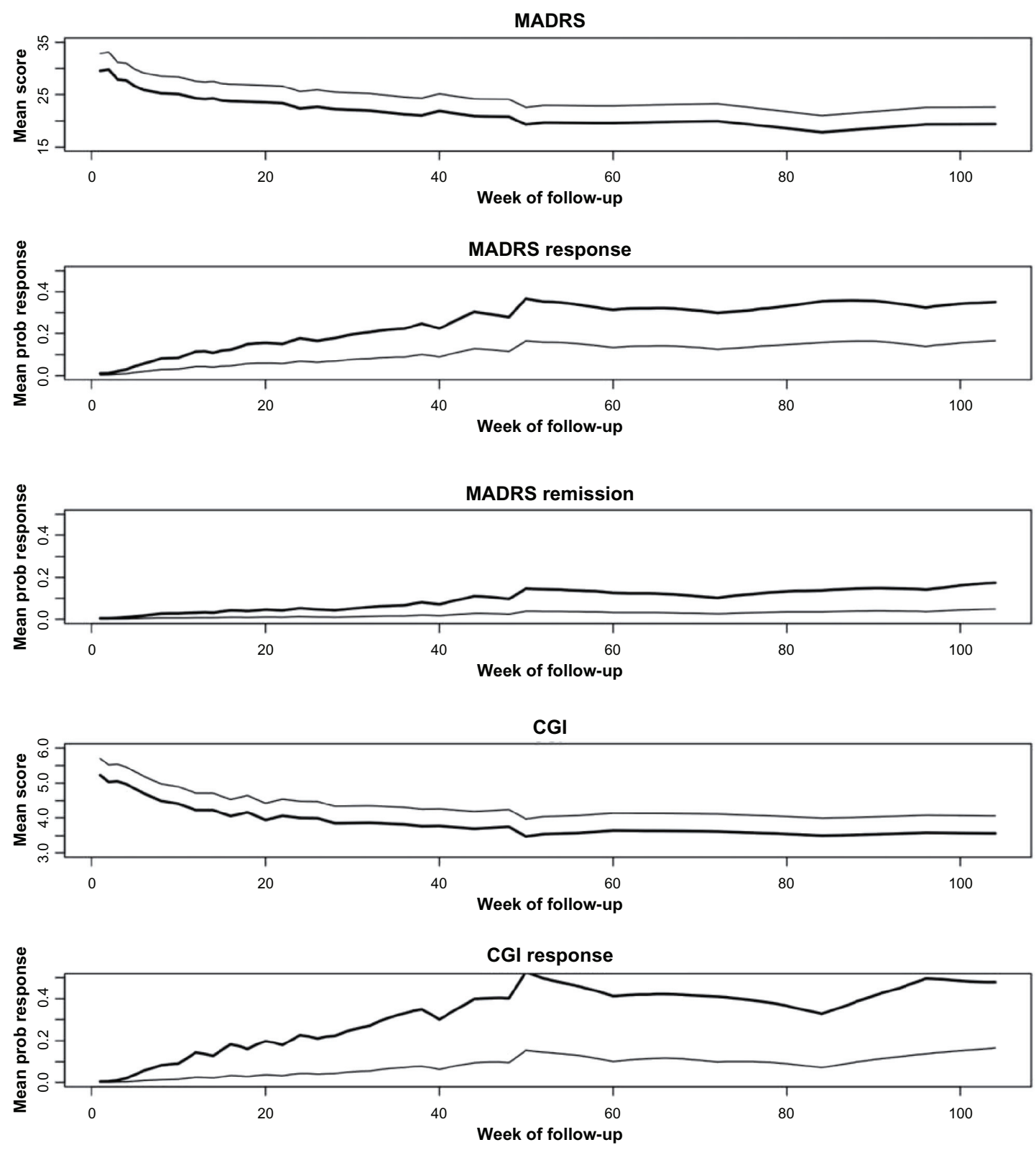

Figure I Mean score or probability of response across week of follow-up for VNS + TAU (dark line) and TAU alone (light line).

Abbreviations: CGI, Clinical Global Impressions scale's Improvement subscale; MADRS, Montgomery-Åsberg Depression Rating Scale; TAU, treatment as usual; VNS, vagus nerve stimulation.

within 2 weeks of each other were combined for the purposes of presentation. Graphically, there is a trend toward decreasing MADRS and CGI-I score associated with VNS + TAU therapy over the course of follow-up.

Results from the repeated measures models indicate that patients who received VNS + TAU experienced a greater benefit over 96 weeks of treatment, as measured by both the MADRS and CGI-I, than did patients who received TAU alone (Table 2). Compared to patients who received TAU only, those who received VNS + TAU had lower MADRS scores (mean difference of -3.26 points; $95 \%$ confidence interval
[CI]: $-3.99,-2.54)$, and the odds of a MADRS response in the VNS + TAU group was 3.19 times greater (95\% CI: 2.12, 4.66), and the odds of a MADRS remission was 4.99 times greater $(95 \%$ CI: $2.93,7.76)$. Similarly, patients who received VNS + TAU had lower CGI-I score (mean difference of - 0.49 points; $95 \% \mathrm{CI}:-0.59,-0.39$ ) and had seven times the odds of CGI-I response $(95 \%$ CI: $4.63,10.83)$ compared to patients who received TAU alone.

Analyses were repeated within subgroups defined by propensity score quintiles and similar results were obtained (Figure S3). 
Table 2 Summary of repeated measures analysis

\begin{tabular}{llllll}
\hline Model-estimated treatment effect & CGI-I & & & MADRS \\
\cline { 2 - 3 } \cline { 5 - 6 } & Estimate & $\mathbf{9 5 \%} \mathbf{C l}$ & & Estimate & $\mathbf{9 5 \%} \mathbf{C l}$ \\
\hline Average difference in scores: VNS + TAU vs TAU & -0.49 & -0.59 to -0.39 & & -3.26 & -3.99 to -2.54 \\
Odds ratio of response: VNS + TAU vs TAU & 7.00 & 4.63 to 10.83 & & 3.19 & 2.12 to 4.66 \\
Odds ratio of remission: VNS + TAU vs TAU & N/A & N/A & & 4.99 & 2.93 to 7.76 \\
\hline
\end{tabular}

Abbreviations: CGI-I, Clinical Global Impressions-Improvement; Cl, confidence interval; MADRS, Montgomery-Åsberg Depression Rating Scale; TAU, treatment as usual; VNS, vagus nerve stimulation; NA, not applicable.

\section{Sustained response}

Overall, among patients who received TAU, 59 patients were considered MADRS responders at 24 weeks and 33 of these 59 patients $(56 \%)$ also had a sustained response at 48 weeks. In the TAU group, 21 patients were also evaluated for MADRS at 96 weeks, and 10 (48\%) had achieved a sustained response at 96 weeks (Table 3). Among patients who received VNS + TAU, 217 patients achieved MADRS response at 24 weeks and 153 of these 217 patients (71\%) had a sustained response at 48 weeks. Additionally, of the 104 patients in the VNS + TAU group evaluated at 96 weeks, $70(67 \%)$ had a sustained MADRS response.

The odds ratio for sustained response for patients who had responded to VNS + TAU at 24 weeks versus TAU patients was 1.98 (95\% CI: $1.34,3.01)$ at 48 weeks and was
3.42 (95\% CI: $1.78,7.31)$ at 96 weeks. That is, patients treated with VNS + TAU who do achieve a response have a greater likelihood of sustaining the response over time. A scatter plot of the pairwise percent changes from baseline in MADRS score at 24, 48, and 96 weeks is presented as Figure 2.

Results were similar for sustained MADRS remission, and sustained CGI-I response. Table 3 shows the number and proportion of patients who achieved MADRS response, MADRS remission, and CGI-I response at 48 and 96 weeks tabulated by response at 24 weeks. The odds ratio for VNS + TAU versus TAU alone for sustained MADRS remission was $2.73(95 \% \mathrm{CI}: 1.49,5.54)$ at 48 weeks and 2.64 (95\% CI: 1.16, 7.19) at 96 weeks. The odds ratio for sustained CGI-I response was 3.09 (95\% CI: $2.09,4.70)$ at 48 weeks and 7.04 (95\% CI: 3.39, 17.27) at 96 weeks.

Table 3 Sustained response and remission rates (in bold) at week 48 and 96 by response or remission status at week 24

\begin{tabular}{|c|c|c|c|c|}
\hline & \multicolumn{2}{|c|}{ Status at 24 weeks for VAS + TAU } & \multicolumn{2}{|c|}{ Status at 24 weeks for TAU } \\
\hline & Non response & Response & Non response & Response \\
\hline \multicolumn{5}{|c|}{ MADRS response } \\
\hline \multicolumn{5}{|c|}{48 weeks } \\
\hline Non response & $410(75 \%)$ & $64(29 \%)$ & 197 (83\%) & $26(44 \%)$ \\
\hline Response & 137 (25\%) & I53 (7 I\%) & $39(17 \%)$ & $33(56 \%)$ \\
\hline \multicolumn{5}{|l|}{96 weeks } \\
\hline Non response & 146 (70\%) & $34(33 \%)$ & $95(86 \%)$ & II (52\%) \\
\hline Response & $64(30 \%)$ & 70 (67\%) & $15(14 \%)$ & 10 (48\%) \\
\hline \multicolumn{5}{|l|}{ CGI-I response } \\
\hline \multicolumn{5}{|l|}{48 weeks } \\
\hline Non response & $378(69 \%)$ & $47(19 \%)$ & $223(86 \%)$ & $23(43 \%)$ \\
\hline Response & $173(31 \%)$ & $204(81 \%)$ & $36(14 \%)$ & 31 (57\%) \\
\hline \multicolumn{5}{|l|}{96 weeks } \\
\hline Non response & 140 (64\%) & $30(25 \%)$ & 91 (72\%) & 14 (67\%) \\
\hline \multirow[t]{2}{*}{ Response } & 78 (36\%) & $90(75 \%)$ & $34(27 \%)$ & 7 (33\%) \\
\hline & Non remission & Remission & Non remission & Remission \\
\hline \multicolumn{5}{|c|}{ MADRS Remission } \\
\hline \multicolumn{5}{|c|}{48 weeks } \\
\hline Non remission & $564(86 \%)$ & 37 (33\%) & $249(92 \%)$ & 14 (56\%) \\
\hline Remission & $89(14 \%)$ & $74(67 \%)$ & $21(8 \%)$ & I I (44\%) \\
\hline \multicolumn{5}{|l|}{96 weeks } \\
\hline Non remission & 208 (8I\%) & 21 (37\%) & II 0 (92\%) & $5(45 \%)$ \\
\hline Remission & 49 (19\%) & $36(63 \%)$ & $10(8 \%)$ & $6(55 \%)$ \\
\hline
\end{tabular}

Note: Bold font highlights numbers (and percentages) of patients with response or remission at week 24 who sustained response or remission at weeks 48 and 96 . Abbreviations: CGI-I, Clinical Global Impressions-Improvement; MADRS, Montgomery-Åsberg Depression Rating Scale; TAU, treatment as usual; VNS, vagus nerve stimulation. 
A

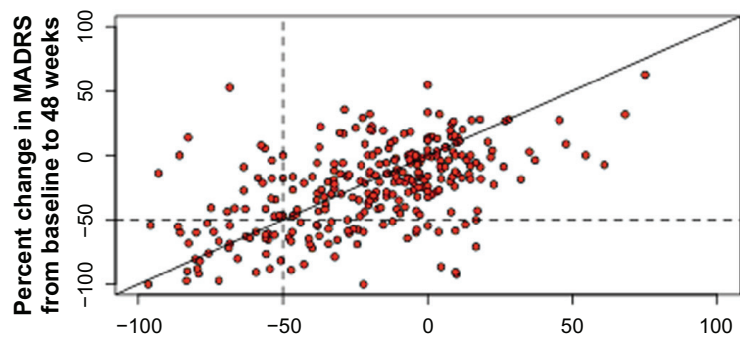

Percent change in MADRS from baseline to 24 weeks

C

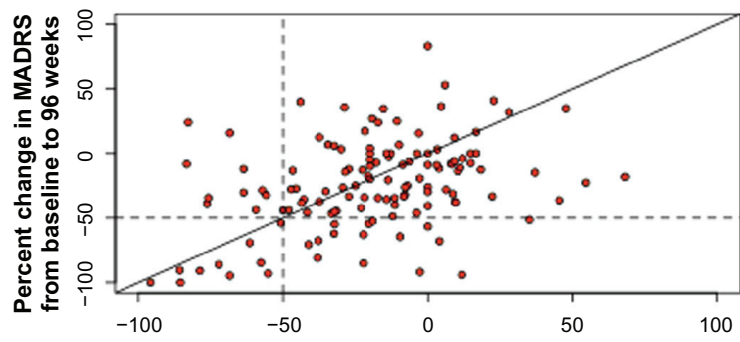

Percent change in MADRS from baseline to 24 weeks

E

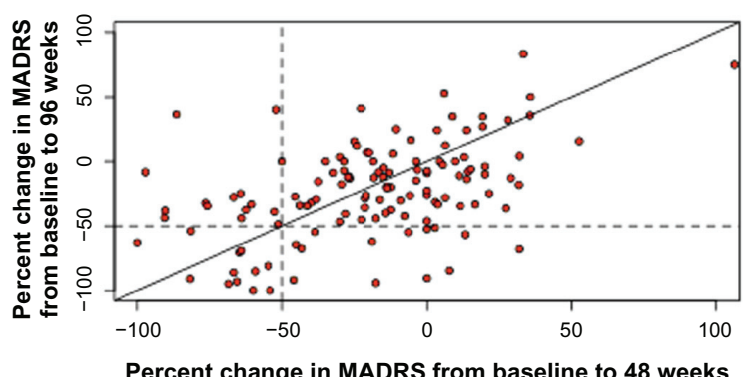

B

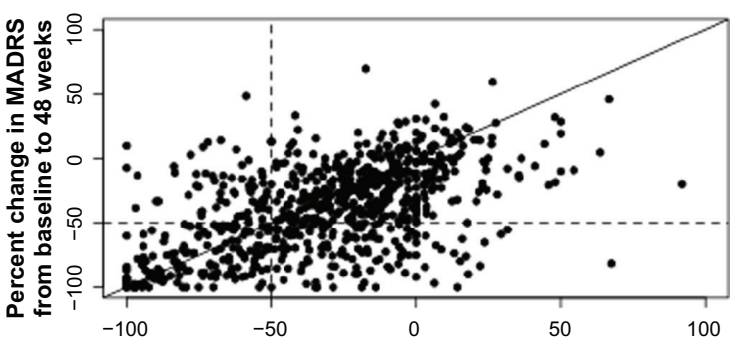

Percent change in MADRS from baseline to 24 weeks

D

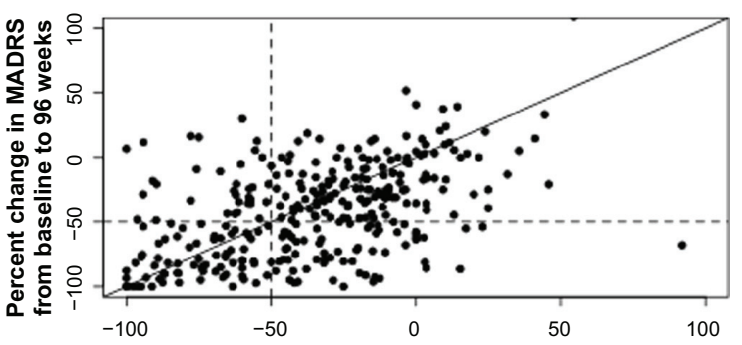

Percent change in MADRS from baseline to 24 weeks

$\mathbf{F}$

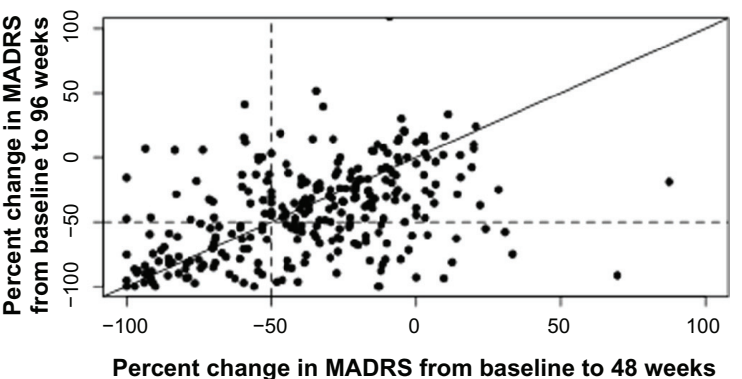

Figure 2 Scatter plot of pairwise percent changes in MADRS from baseline over time. (A and B) Change from 24 to 48 weeks; (C and D) change from 24 to 96 weeks; (E and F) change from 48 to 96 weeks.

Note: Red, TAU; black, VNS + TAU. Patients falling into the -50 to -100 quadrant at 48 and 96 weeks are considered sustained responders.

Abbreviations: MADRS, Montgomery-Åsberg Depression Rating Scale; TAU, treatment as usual; VNS, vagus nerve stimulation.

\section{NNT}

To provide a sense of the magnitude of the effects of VNS + TAU, we examined the NNT for VNS using observed response rates (Table 4). ${ }^{31}$ After 12 weeks of treatment, the NNT for VNS + TAU was calculated to be $8(95 \%$ CI: 6,12$)$, and the NNT decreased over time to $7(95 \%$ CI: 5, 12), 6 (95\% CI: 4, 9), and 4 (95\% CI: 3, 6) at 24, 48, and 96 weeks, respectively.

From the acute phase, randomized, controlled trials comparing ECT to tricyclic antidepressant (TCA), the NNT was 5 (95\% CI: 2, 2676) and the NNT for ECT to monoamine oxidase inhibitors (MAOI) was 3 (95\% CI: 2, 3). ${ }^{32}$ NNTs based on seven nonrandomized studies of ECT versus antidepressants (TCAs, MAOIs, selective serotonin reuptake inhibitors [SSRIs], and lithium + TCA) were considered better comparators with the present meta-analysis of VNS + TAU, and the NNT from nonrandomized trials comparing ECT to antidepressants was 4 (95\% CI: 3 to 9$).{ }^{32}$

From a randomized, double-blind, controlled trial comparing patients who had received open-label ECT and were subsequently randomized to receive continuation treatment for 24 weeks with placebo, nortriptyline, or nortriptyline/ lithium; the NNT for ECT + nortriptyline versus placebo was 4 (95\% CI: 2, 83) and the NNT for ECT + nortriptyline/ lithium versus placebo was 2 (95\% CI: 1 to 4$).{ }^{33}$

\section{Safety assessment}

AEs were only collected in Studies D-01, D-02, D-03, and D-21, and, therefore, no comparative AE data are available on the TAU population. As VNS is an adjunctive treatment, the VNS Therapy patients were also receiving other antidepressant treatments during study participation. 
Table 4 Numbers needed to treat for benefit for ECT (versus controls) and for VNS + TAU (versus TAU)

\begin{tabular}{|c|c|c|}
\hline & $\begin{array}{l}\text { Number } \\
\text { needed } \\
\text { to treat } \\
\text { (for benefit) }\end{array}$ & $\begin{array}{l}95 \% \\
\text { confidence } \\
\text { interval }\end{array}$ \\
\hline \multicolumn{3}{|l|}{ Acute studies } \\
\hline RCT (ECT vs TCA control) $)^{3 !}$ & 5 & 2 to 2676 \\
\hline RCT (ECT vs MAOI control) $)^{31}$ & 3 & 2 to 3 \\
\hline Non-RCT (ECT vs AD ${ }^{\mathrm{a}}$ control) $)^{31}$ & 4 & 3 to 9 \\
\hline At 12 weeks, VNS + TAU vs TAU & 8 & 6 to 12 \\
\hline \multicolumn{3}{|c|}{ Week 24 NNTs from 24-week long studies } \\
\hline $\mathrm{ECT}+$ nortriptyline vs placebo control ${ }^{32}$ & 4 & 2 to 83 \\
\hline $\begin{array}{l}\text { ECT + nortriptyline/lithium } \\
\text { vs placebo control }{ }^{32}\end{array}$ & 2 & I to 4 \\
\hline VNS + TAU vs TAU & 7 & 5 to 12 \\
\hline \multicolumn{3}{|c|}{ Week 48 and 96 NNTs from 48 and 96 -week long studies } \\
\hline At 48 weeks, VNS + TAU vs TAU & 6 & 4 to 9 \\
\hline At 96 weeks, VNS + TAU vs TAU & 4 & 3 to 6 \\
\hline
\end{tabular}

Note: ${ }^{a}$ Antidepressants included tricyclic antidepressants, monoamine oxidase inhibitors, selective serotonin reuptake inhibitors, and lithium.

Abbreviations: AD, antidepressants; ECT, electroconvulsive therapy; MAOI, monoamine oxidase inhibitors; RCT, randomized clinical trial; TAU, treatment as usual; TCA, tricyclic antidepressant; VNS, vagus nerve stimulation.

Overall, VNS was well tolerated. The withdrawal rates during the combined acute and long-term phases were comparable between the treatment groups $(23.6 \%$ for $\mathrm{VNS}+\mathrm{TAU}$ versus $22.2 \%$ for TAU). The most commonly cited reasons for withdrawal were: patient withdrew consent $(3.4 \%$ for VNS + TAU versus $6.3 \%$ for TAU), protocol noncompliance $(2.1 \%$ for VNS + TAU versus $4.9 \%$ for TAU), and lack of efficacy ( $2.3 \%$ for $\mathrm{VNS}+\mathrm{TAU}$ versus $0 \%$ for TAU). Unspecified reasons (or other) accounted for withdrawal of $15.8 \%$ from the VNS + TAU group versus $11.0 \%$ from the TAU group.

The number (and percentage) of patients reporting an $\mathrm{AE}$ during the first and second years on VNS Therapy is provided in Table S4. The table lists AEs that were reported in $\geq 10 \%$ of total patients in the first year following implantation. Note that each patient is counted only once per $\mathrm{AE}$ within each year even though some patients may have reported multiple occurrences of the same event. As expected, the most common AEs were voice alteration, dyspnea, pain, cough increased, and incision pain (Table S4). These events are similar to what has been previously reported in the literature. ${ }^{16,19-25}$ Notably, there is a trend towards diminishing AEs over the 2 years of treatment with VNS; and such a trend is consistent with previous findings from VNS studies in patients with epilepsy. ${ }^{34}$

\section{Discussion}

Bayesian hierarchical modeling was used to compare scores (using the MADRS and CGI-I), response rates (using the
MADRS and CGI-I), and remission rates (using the MADRS) following treatment with VNS + TAU versus TAU in a chronic TRD population. Outcomes were compared over time (up to 96 weeks of treatment) in a sample of over 1000 patients in the VNS + TAU group and over 400 patients in the TAU group from six prospective clinical studies evaluating VNS + TAU or TAU. This Bayesian meta-analysis found a consistent superiority of VNS + TAU over TAU.

Compared with outpatients evaluated in the Sequenced Treatment Alternatives to Relieve Depression (STAR*D) study who had not responded to three to four treatment steps, ${ }^{5}$ patients in this meta-analysis were more likely to be Caucasian and to have had a longer length of illness, a higher suicide attempt rate, and more previous depression treatments, including ECT. Thus, the chronic treatment resistant nature of the VNS + TAU patients is indisputable. Nevertheless, the odds of treatment response in VNS + TAU patients were three times that for patients who received TAU alone, and the odds of remission in VNS + TAU patients were almost five times as likely as that for TAU patients (Table 2).

As this meta-analysis included all company-sponsored completed and ongoing multicenter VNS Therapy studies, there is no additional published literature examining VNS + TAU versus TAU against which to compare these findings directly. However, the response rates in the $\mathrm{VNS}+\mathrm{TAU}$ patients are comparable in magnitude to those obtained with ECT, which is known to be superior to pharmacotherapy in treating patients with severe or difficult to treat depression. ${ }^{35}$ Meta-analyses of ECT trials in MDD have shown similar superiority of response, which was approximately three times greater than with simulated ECT (odds ratio: 2.83 ; CI: $1.30,6.17)$, and approximately four times greater for ECT in comparison to antidepressants (TCAs, MAOIs, SSRIs, and lithium + TCA), in a clinical setting (odds ratio: 3.72; CI: 2.60, 5.32). ${ }^{32}$

To provide additional clinical context for these findings, we compared the NNT over time for VNS versus ECT (Table 4). NNTs from acute phase trials comparing ECT to various anti-depressants were as follows: 5 for ECT versus TCA, and 3 for ECT versus MAOI. For VNS + TAU, the NNT decreases over time from 12 to 96 weeks (NNT of 8 , 7, 6, and 4 at 12, 24, 48, and 96 weeks, respectively). Thus, longer-term VNS + TAU treatment appears to have a similar magnitude of effectiveness as short-term ECT.

We acknowledge that ECT is an effective acute episodic treatment option and that VNS Therapy is not an acute therapy but a potential longer-term approach with sustained treatment delivery. VNS is not meant to replace ECT, and 
there are published reports indicating that both treatments can be used concomitantly. ${ }^{36-38}$

Patients diagnosed with the more difficult to treat bipolar depression have similar effect sizes $^{39}$ to those seen for VNS Therapy treated. ${ }^{16}$ However, the effectiveness of ECT in patients who have not responded to pharmacotherapy is lower (odds ratio: 0.52; CI: 0.39, 0.69); ${ }^{40}$ than for those without prior pharmacotherapy failure. Overall, patients included in this meta-analysis had a mean of about six treatment failures prior to study entry $(5.9 \pm 2.2$ for the TAU group and $6.9 \pm 2.2$ for the VNS + TAU group; Table 1) - which makes them at least as treatment-resistant as those completing the fourth treatment step in the STAR*D study.

If ECT is acutely effective, then sustaining that benefit whether response or remission-over time remains a challenge. In one study, $53 \%$ of ECT patients who achieved remission relapsed within 12 months, with $94 \%$ of those relapses occurring during the first 6 months after a course of ECT. ${ }^{41}$ The rate of relapse was significantly greater, at $68 \%$ in patients who were considered resistant to pharmacotherapy. Continued pharmacotherapy after remitting with ECT treatment can reduce relapse rates; $39 \%$ after 6 months with a combination of nortriptyline and lithium treatment, but the rate is still high. ${ }^{33} \mathrm{VNS}+$ TAU treated patients responding at 24 weeks are $2-4$ times more likely to maintain that response at 48 weeks over TAU (Table 3). This is consistent with previous studies that show over $60 \%$ of the VNS treated patients who responded at either 3 or 12 months maintained that response for up to 24 months. ${ }^{42}$ Although response takes longer to attain with VNS Therapy, it is more likely to be sustained than with ECT treatment where the response is more immediate, but less sustainable.

One of the strengths of this meta-analysis is that it involved the largest database of interventional studies of patients with TRD over a long time period of 2 years, which is important in a chronic disease state like TRD. Further, it represents a culmination of all available multicenter studies with VNS in this patient population, the consistent use of commonly used and validated scales for measuring depression symptomatology, and an analysis performed at the individual patient data level rather than using summary data, as is the case with most meta-analysis studies. The primary limitation of the meta-analysis involves the individual study designs; namely, that the TAU group data is limited to two trials for the CGI-I scale (ie, Studies D-04 and D-23) and one trial for the MADRS scale (Study D-23); in addition, the nonrandomized D-23 study and the randomized, sham-controlled acute phase of the D-02 study represent the only concurrent head-to-head comparisons of VNS + TAU and TAU. We also note that patients included in this study were out-patients with an overall moderately severe to severe disease state but not very severe symptoms (Table 1). The medications that the patients were receiving prior to VNS achieved some stability and modest benefit but had not produced a remission, and the patients continued to have a clinically meaningful level of symptom severity and poor function. Thus, the patients were candidates for a different treatment approach. Finally, the issue of blinding the investigators and patients to VNS. Therapy is difficult for a treatment that has benign - but well characterized and observable - side effects. Discussions between the field of psychiatry and the FDA have been undertaken regarding these multifaceted questions of study design and blinding for devices in the treatment of depression, with limited success. ${ }^{43}$

\section{Conclusion}

This meta-analysis reveals a substantial and sustained difference in both response and remission rates between VNS + TAU and TAU treatment in patients suffering from a treatment-resistant major depressive episode.

\section{Author contributions}

Mr Jayewardene, Dr Bunker, and Dr Olin compiled the raw data, and the demographics and patient disposition data; they also performed the NNT analysis. Dr Berry and Ms Broglio conducted the statistical analysis of the data and Cyberonics, Inc (Houston, TX, USA) compensated their employer (Berry Consultants, Austin, TX, USA). All listed authors were responsible for data interpretation and conclusions.

\section{Acknowledgments}

Medical writing assistance was provided by Karishma Manzur, PhD (employee of Lenimen Consulting, Inc), and was compensated by Cyberonics, Inc (Houston, TX, USA). The authors would also like to acknowledge the late Andrew Leon, $\mathrm{PhD}$ for discussions on statistical methodology that led to improvements in the proposed analysis.

\section{Disclosure}

Cyberonics, Inc (Houston, TX, USA) is the manufacturer of VNS Therapy ${ }^{\circledR}$ and sponsored the studies included in the meta-analysis. Dr Olin, Dr Bunker, and Mr Jayewardene are employees of Cyberonics; in addition, Dr Olin and Dr Bunker are stockholders of Cyberonics. Dr Berry and Ms Broglio are employees of Berry Consultants (Austin, TX, USA), which was commissioned by Cyberonics to perform independent 
statistical analyses. Dr Rush has received consulting fees from Otsuka Pharmaceutical Co, Ltd, University of Michigan, and Brain Resource Ltd; speaker fees from Singapore College of Family Physicians; royalties from Guilford Publications and the University of Texas Southwestern Medical Center; a travel grant from CINP; and research support from the National Institute of Mental Health and Duke-NUS Graduate Medical School, Singapore. The authors report no other conflicts of interest in this work.

\section{References}

1. WHO: Depression [webpage on the Internet]. Geneva: World Health Organization. Available from: http://www.who.int/mediacentre/ factsheets/fs369/en/index.html. Accessed January 24, 2012.

2. Kessler RC, Petukhova M, Sampson NA, Zaslavsky AM, Wittchen HU. Twelve-month and lifetime prevalence and lifetime morbid risk of anxiety and mood disorders in the United States. Int J Methods Psychiatr Res. 2012;21(3):169-184.

3. Greenberg PE, Birnbaum HG. The economic burden of depression in the US: societal and patient perspectives. Expert Opin Pharmacother. 2005;6(3):369-376.

4. Kessler RC. The costs of depression. Psychiatr Clin North Am. 2012;35(1):1-14.

5. Rush AJ, Trivedi MH, Wisniewski SR, et al. Acute and longer-term outcomes in depressed outpatients requiring one or several treatment steps: a STAR*D report. Am J Psychiatry. 2006;163(11):1905-1917.

6. Schachter SC, Schmidt D. Vagus Nerve Stimulation. London, England: Martin Dunitz; 2001.

7. Ben-Menachem E, Hellstrom K, Verstappen D. Analysis of direct hospital costs before and 18 months after treatment with vagus nerve stimulation therapy in 43 patients. Neurology. 24 2002;59(6 Suppl 4): S44-S47.

8. Boon P, D'Have M, Van Walleghem P, et al. Direct medical costs of refractory epilepsy incurred by three different treatment modalities: a prospective assessment. Epilepsia. 2002;43(1):96-102.

9. Bernstein AL, Hess T. Vagus nerve stimulation therapy for pharmacoresistant epilepsy: effect on health care utilization. Epilepsy Behav. 2007;10(1):134-137.

10. Helmers SL, Duh MS, Guerin A, et al. Clinical and economic impact of vagus nerve stimulation therapy in patients with drug-resistant epilepsy. Epilepsy Behav. 2011;22(2):370-375.

11. Helmers SL, Duh MS, Guerin A, et al. Clinical outcomes, quality of life, and costs associated with implantation of vagus nerve stimulation therapy in pediatric patients with drug-resistant epilepsy. Eur $J$ Paediatr Neurol. 2012;16(5):449-458.

12. Cohen LJ, Allen JC Jr. Estimating the potential savings with vagus nerve stimulation for treatment-resistant depression: a payer perspective. $\mathrm{Curr}$ Med Res Opin. 2008;24(8):2203-2217.

13. Feldman RL, Dunner DL, Muller JS, Stone DA. Medicare patient experience with vagus nerve stimulation for treatment-resistant depression. J Med Econ. 2013;16(1):62-74.

14. Martin JL, Martin-Sanchez E. Systematic review and meta-analysis of vagus nerve stimulation in the treatment of depression: variable results based on study designs. Eur Psychiatry. 2012;27(3):147-155.

15. Daban C, Martinez-Aran A, Cruz N, Vieta E. Safety and efficacy of Vagus Nerve Stimulation in treatment-resistant depression. A systematic review. J Affect Disord. 2008;110(1-2):1-15.

16. Aaronson ST, Carpenter LL, Conway CR, et al. Vagus nerve stimulation therapy randomized to different amounts of electrical charge for treatment-resistant depression: acute and chronic effects. Brain Stimul. Epub October 23, 2012.

17. Montgomery SA, Asberg M. A new depression scale designed to be sensitive to change. Br J Psychiatry. 1979;134:382-389.
18. Guy W. ECDEU Assessment Manual for Psychopharmacology. Rockville, MD: US Department of Health, Education, and Welfare; 1976.

19. Rush AJ, George MS, Sackeim HA, et al. Vagus nerve stimulation (VNS) for treatment-resistant depressions: a multicenter study. Biol Psychiatry. 2000;47(4):276-286.

20. Nahas Z, Marangell LB, Husain MM, et al. Two-year outcome of vagus nerve stimulation (VNS) for treatment of major depressive episodes. J Clin Psychiatry. 2005;66(9):1097-1104.

21. Sackeim HA, Rush AJ, George MS, et al. Vagus nerve stimulation (VNS) for treatment-resistant depression: efficacy, side effects, and predictors of outcome. Neuropsychopharmacology. 2001;25(5):713-728.

22. Rush AJ, Marangell LB, Sackeim HA, et al. Vagus nerve stimulation for treatment-resistant depression: a randomized, controlled acute phase trial. Biol Psychiatry. 2005;58(5):347-354.

23. Rush AJ, Sackeim HA, Marangell LB, et al. Effects of 12 months of vagus nerve stimulation in treatment-resistant depression: a naturalistic study. Biol Psychiatry. 2005;58(5):355-363.

24. Schlaepfer TE, Frick C, Zobel A, et al. Vagus nerve stimulation for depression: efficacy and safety in a European study. Psychol Med.2008; 38(5):651-661.

25. Bajbouj M, Merkl A, Schlaepfer TE, et al. Two-year outcome of vagus nerve stimulation in treatment-resistant depression. $J$ Clin Psychopharmacol. 2010;30(3):273-281.

26. Dunner DL, Rush AJ, Russell JM, et al. Prospective, long-term, multicenter study of the naturalistic outcomes of patients with treatmentresistant depression. J Clin Psychiatry. 2006;67(5):688-695.

27. George MS, Rush AJ, Marangell LB, et al. A one-year comparison of vagus nerve stimulation with treatment as usual for treatment-resistant depression. Biol Psychiatry. 2005;58(5):364-373.

28. Guidelines for ATC Classification and DDD Assignment 2011. Oslo: World Health Organization (WHO) Collaborating Centre for Drug Statistics Methodology; 2010. Available from: http://www.whocc.no/filearchive/ publications/2011guidelines.pdf. Accessed 24 January 2012.

29. Berry SM, Carlin BP, Lee JJ, Muller P. Bayesian Adaptive Methods for Clinical Trials. Vol 38. Boca Raton, FL: CRC Press; 2010.

30. West M, Harrison J. Bayesian Forecasting and Dynamic Models. New York, NY: Springer-Verlag; 1977.

31. McQuay HJ, Moore RA. Using numerical results from systematic reviews in clinical practice. Ann Intern Med. 1997;126(9): 712-720.

32. Pagnin D, de Queiroz V, Pini S, Cassano GB. Efficacy of ECT in depression: a meta-analytic review. $J E C T$. 2004;20(1):13-20.

33. Sackeim HA, Haskett RF, Mulsant BH, et al. Continuation pharmacotherapy in the prevention of relapse following electroconvulsive therapy: a randomized controlled trial. JAMA. 2001;285(10):1299-1307.

34. Morris GL 3rd, Mueller WM. Long-term treatment with vagus nerve stimulation in patients with refractory epilepsy. The Vagus Nerve Stimulation Study Group E01-E05. Neurology. 1999;53(8): 1731-1735.

35. UK ECT Review Group. Efficacy and safety of electroconvulsive therapy in depressive disorders: a systematic review and meta-analysis. Lancet. March 8, 2003;361(9360):799-808.

36. Santermans L, Vanderbruggen N, Zeeuws D, Baeken C. Successful ECT treatment after relapse during VNS therapy. Psychiatr Danub. 2010;22 Suppl 1:S166.

37. Warnell RL, Elahi N. Introduction of vagus nerve stimulation into a maintenance electroconvulsive therapy regimen: a case study and cost analysis. $J E C T$. 2007;23(2):114-119.

38. Burke MJ, Husain MM. Concomitant use of vagus nerve stimulation and electroconvulsive therapy for treatment-resistant depression. J ECT. 2006;22(3):218-222.

39. Dierckx B, Heijnen WT, van den Broek WW, Birkenhager TK. Efficacy of electroconvulsive therapy in bipolar versus unipolar major depression: a meta-analysis. Bipolar Disord. 2012;14(2): $146-150$. 
40. Heijnen WT, Birkenhager TK, Wierdsma AI, van den Broek WW. Antidepressant pharmacotherapy failure and response to subsequent electroconvulsive therapy: a meta-analysis. J Clin Psychopharmacol. 2010;30(5):616-619.

41. Sackeim HA, Prudic J, Devanand DP, et al. A prospective, randomized, double-blind comparison of bilateral and right unilateral electroconvulsive therapy at different stimulus intensities. Arch Gen Psychiatry. 2000;57(5):425-434.

42. Sackeim HA, Brannan SK, Rush AJ, George MS, Marangell LB, Allen J. Durability of antidepressant response to vagus nerve stimulation (VNS) Int J Neuropsychopharmacol. 2007;10(6):817-826.
43. October 8, 2010: Neurological Devices Panel Meeting FDA Generated Materials. Silver Spring, MD: US Food and Drug Administration; 2010. Available from: http://www.fda.gov/AdvisoryCommittees/CommitteesMeetingMaterials/MedicalDevices/MedicalDevicesAdvisoryCommittee/NeurologicalDevicesPanel/ucm202072.htm. Accessed January 24, 2012.

44. CST95 - Coding Symbols for Thesaurus of Adverse Reaction Terms (COSTART). 5th ed. Rockville, MD: U.S. Food and Drug Administration; Center for Drug Evaluation and Research; 1995. 


\section{Supplementary materials}

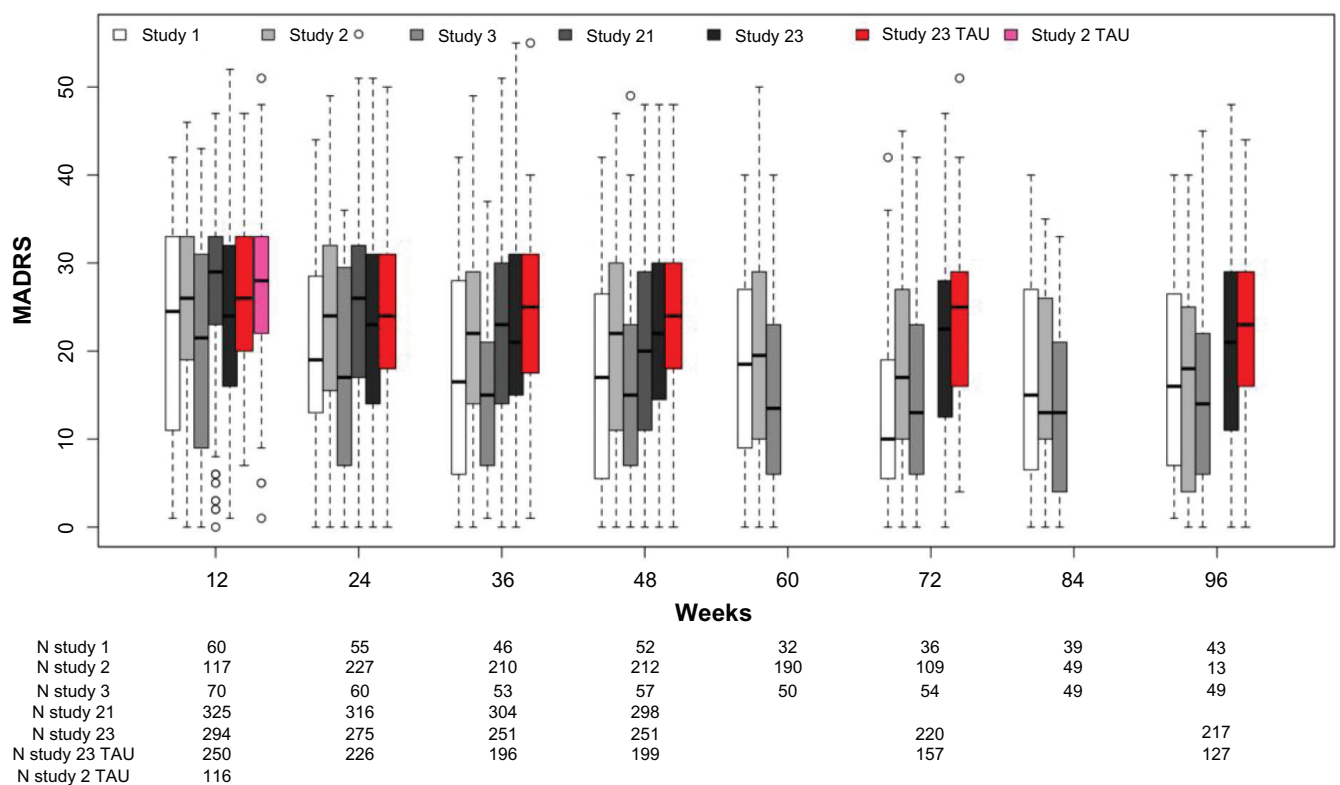

Figure SI Box and whisker plots of the MADRS score by study, week of follow-up, and treatment group.

Notes: Box is defined by the 75th percentile (top), the median (middle), and the 25th percentile (bottom). The whiskers extend to the greater of I.5 times the inner-quartile range (box size) or the maximum and minimum values. Outliers are individual data points.

Abbreviations: MADRS, Montgomery-Åsberg Depression Rating Scale; TAU, treatment as usual.

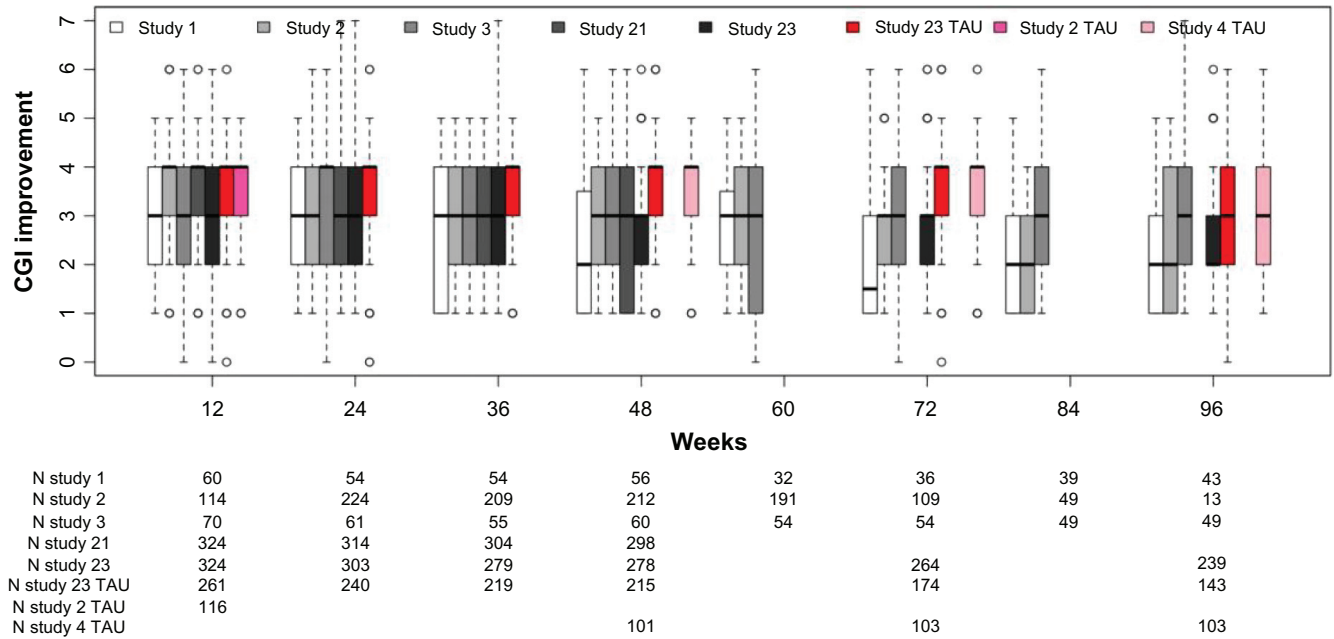

Figure S2 Box and whisker plots of the CGI Improvement score by study, week of follow-up, and treatment group.

Notes: Box is defined by the 75th percentile (top), the median (middle), and the 25 th percentile (bottom). The whiskers extend to the greater of I.5 times the inner-quartile range (box size) or the maximum and minimum values. Outliers are individual data points.

Abbreviations: CGI, Clinical Global Impressions scale; TAU, treatment as usual. 
A

CGI

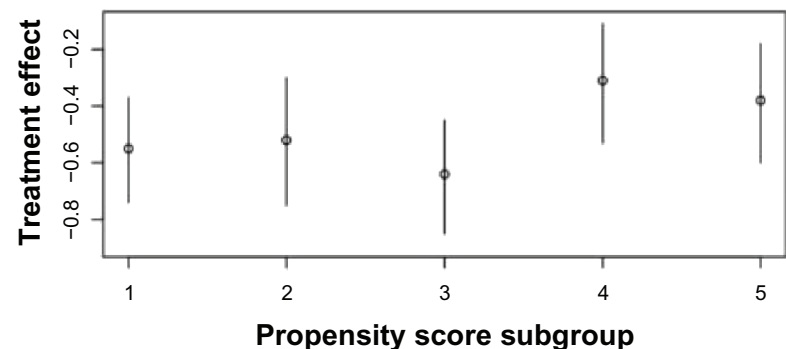

C

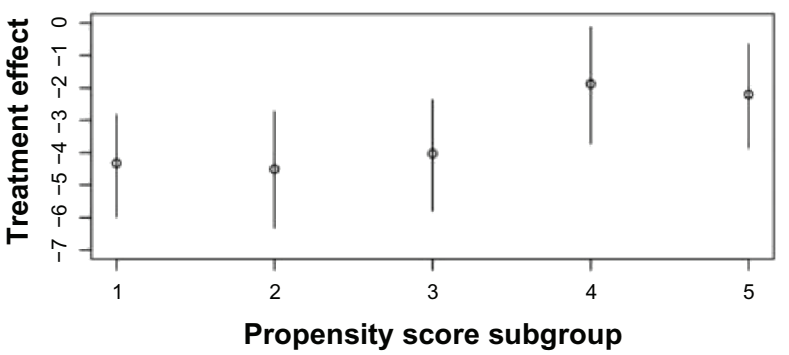

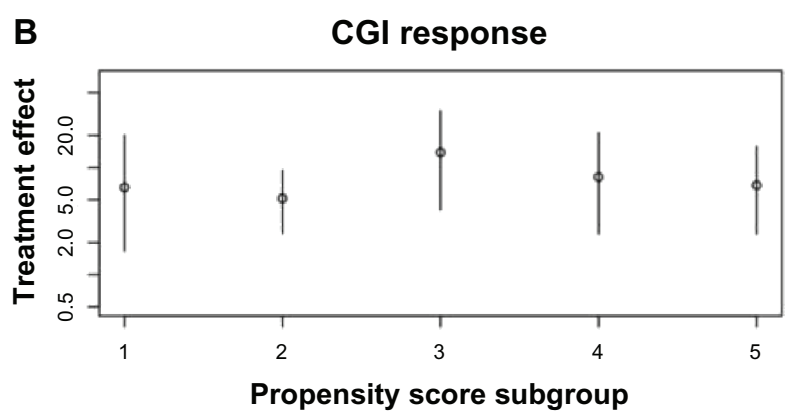

D MADRS response

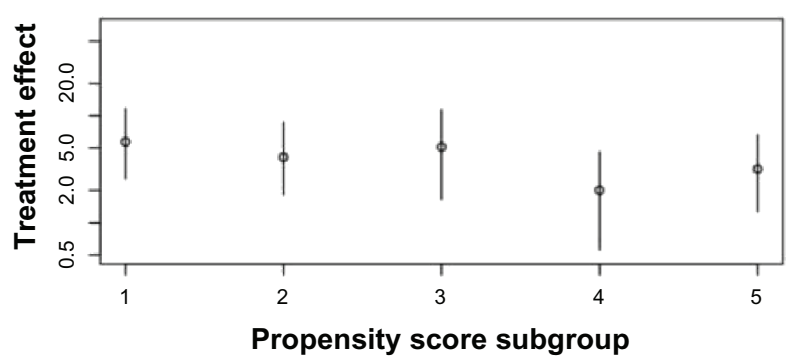

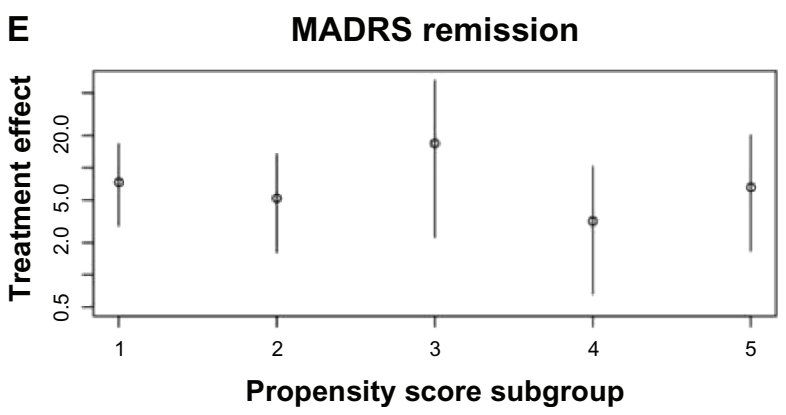

Figure S3 Treatment effects as measured by CGI Improvement and MADRS. (A) CGI; (B) CGI response; (C) MADRS; (D) MADRS response; (E) MADRS remission. Note: $95 \%$ confidence intervals by propensity score group for each endpoint are displayed.

Abbreviations: CGI, Clinical Global Impressions scale; MADRS, Montgomery-Åsberg Depression Rating Scale. 
Table SI Various outcome measures utilized in the six clinical studies included in the meta-analysis

\section{Inclusion criteria}

- Adult male and female patients with a diagnosis of a major depressive episode (unipolar or bipolar disorder) according to DSM-IV Diagnosis Criteria.

- Age requirements: 18 to 70 years of age in D-0I; 18 to 80 years of age in D-02, D-03, and D-04; and >I8 years of age in D-2I and D-23.

- Patient has a history of chronic ( $\geq 2$ years) or recurrent (at least 4 episodes for D-0I, D-02, D-03, and D-04; at least 2 episodes for D-2I and D-23) major depression.

- An inadequate response to antidepressant treatments from at least 2 different treatment categories. Inadequate response was defined as follows:

O D-01, D-02, D-03, and D-04 only: antidepressant resistance rating $\geq 3$ for at least 2 treatments.

O D-2I and D-23 only: resistant to $\geq 4$-adequate antidepressant treatments.

- Patient is able to comply with all testing and visit requirements per protocol.

- D-02, D-03, and D-04 only: a score $\geq 20$ on the 24-Item Hamilton Rating Scale for Depression (HRSD).

- D-0I only: A score $\geq 20$ on the 28-item HRSD.

- D-0I, D-02, and D-03 only: patient is a male or non-pregnant female adequately protected from conception.

- D-2I only: a minimum baseline score of 24 on the Montgomery-Asberg Depression Rating Scale (MADRS) and no greater than a $25 \%$ decrease in the MADRS score between the prestudy and baseline visits.

- D-23 only: a Clinical Global Impression (CGI) severity of illness score of moderately ill (score of $\geq 4$ ).

- D-0I only: patient has a score $\leq 50$ on the Global Assessment of function.

- D-0I, D-02, and D-03 only: stable on current antidepressant medication regimen for $\geq 4$ weeks prior to visit I or not taking antidepressant medications.

- D-2I only: on stable anti-depressant for $>4$ weeks.

- D-0I, D-02, D-03, D-04, and D-2I only: patient with a bipolar disorder has demonstrated a resistance to lithium treatment or has a medical contraindication to treatment with lithium or is known to be intolerant to lithium.

- D-0I, D-02, D-03, and D-04 only: patient has an intelligence quotient (IQ) $\geq 70$ based on the investigator's judgement

- D-02 only: if currently taking atypical antipsychotics, stable medications for $>4$ weeks.

- D-03 only: patient has a history of psychotherapy treatment that did not result in clinical improvement.

- D-2I only: patient with a current diagnosis of bipolar disorder must be receiving a mood stabilizer.

\section{Exclusion criteria}

- A history of schizophrenia, schizoaffective disorder, any other psychotic disorder, or a current major depressive episode that includes psychotic features; or is currently psychotic.

- Other exclusion criteria included simultaneous enrollment in another investigational trial, previously received Vagus Nerve Stimulation therapy, presence of other cognitive disorders (such as delirium or dementia), other neurological problems (such as central nervous system disease or injury), current alcohol or substance abuse, clinically significant suicidal intent, and cardiac or pulmonary disorders (such as history of myocardial infarction or cardiac arrest).

- D-02, D-03, D-04, D-2I, and D-23 only: a history of rapid cycling bipolar disorder.

- D-0I, D-02, D-03, D-2I only: has had a vagotomy.

- D-0I, D-02, D-03, and D-2I only: patient requires a whole body MRI subsequent to VNS implantation.

- D-0I, D-02, and D-2I only: a history of ulcers including gastric and duodenal, vasovagal syncope, or swallowing difficulties.

- D-2I only: a history of borderline personality disorder. 
Table S2 Various outcome measures utilized in the six clinical studies included in the meta-analysis

\begin{tabular}{|c|c|c|c|c|c|c|}
\hline & D-0I & D-02 & D-03 & D-04 & D-2 I & D-23 \\
\hline Hamilton Rating Scale for Depression (HRSD)a & $\mathrm{X}$ & $\mathrm{X}$ & $\mathrm{X}$ & $x^{b}$ & N/A & N/A \\
\hline Montgomery-Åsberg Depression Rating Scale (MADRS) & $\mathrm{X}$ & $x$ & $x$ & $\mathrm{~N} / \mathrm{A}$ & $\mathrm{x}$ & $\mathrm{x}$ \\
\hline Inventory for Depressive Symptomatology-Clinician (IDS-C) & $\mathrm{N} / \mathrm{A}$ & N/A & $\mathrm{N} / \mathrm{A}$ & N/A & $x$ & N/A \\
\hline Inventory for Depressive Symptomatology-Self-Rated (IDS-SR) & N/A & $\mathrm{x}$ & $\mathrm{x}$ & $\mathrm{x}$ & $\mathrm{x}$ & QIDSc \\
\hline $\begin{array}{l}\text { Clinical Global Impressions-Severity (CGI-S) and Clinical Global } \\
\text { Impressions-Improvement (CGI-I) }\end{array}$ & $\mathrm{x}$ & $\mathrm{x}$ & $\mathrm{x}$ & $\mathrm{x}$ & $\mathrm{x}$ & $\mathrm{X}$ \\
\hline
\end{tabular}

Notes: ${ }^{\text {TTh }} 28$ item HRSD scale was used in D-0I and the 24-item scale was used in D-02, D-03, and D-04; b the HRSD was collected only at baseline, 12 months, and 24 months in the D-04 study; 'study D-23 utilized the Quick Inventory for Depressive Symptomatology (QIDS).

Abbreviation: N/A, not applicable. 


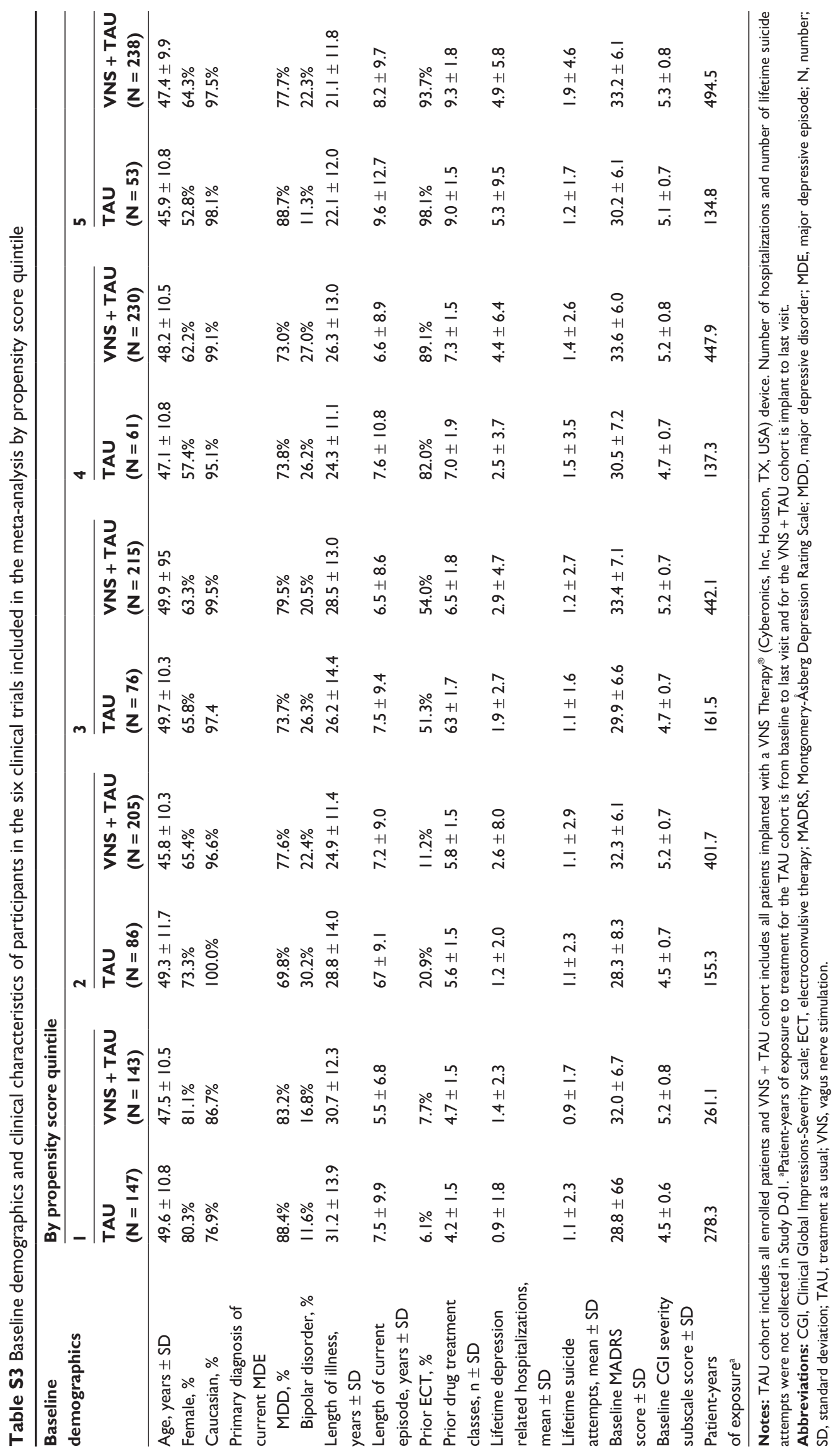


Table S4 Adverse events reported during the first and second years on VNS Therapy ${ }^{\circledR}$ (Cyberonics, Inc, Houston, TX, USA)

\begin{tabular}{|c|c|c|}
\hline Adverse event, n (\%) & $\begin{array}{l}\text { Year I } \\
(N=700)\end{array}$ & $\begin{array}{l}\text { Year } 2 \\
(N=344)\end{array}$ \\
\hline Voice alteration & 485 (69.29) & $179(52.03)$ \\
\hline Dyspnea & $211(30.14)$ & 71 (20.64) \\
\hline Pain & $199(28.43)$ & $4 \mid(11.92)$ \\
\hline Increased cough & I $85(26.43)$ & $47(13.66)$ \\
\hline Incision pain & I8I (25.86) & I5 (4.36) \\
\hline Paresthesia & $159(22.7 I)$ & $39(11.34)$ \\
\hline Headache & $153(21.86)$ & $29(8.43)$ \\
\hline Neck pain & $139(19.86)$ & 55 (I5.99) \\
\hline Pharyngitis & $122(17.43)$ & $25(7.27)$ \\
\hline Depression & I2I (I7.29) & $46(13.37)$ \\
\hline Dysphagia & $115(16.43)$ & $32(9.30)$ \\
\hline Incision-site reaction & $113(16.14)$ & $15(4.36)$ \\
\hline Nausea & $107(15.29)$ & $12(3.49)$ \\
\hline Device-site pain & $98(14.00)$ & II (3.20) \\
\hline Hypertonia & $92(13.14)$ & $31(9.01)$ \\
\hline Device-site reaction & $82(\mid I .7 I)$ & $27(7.85)$ \\
\hline Insomnia & 75 (I0.7I) & $22(6.40)$ \\
\hline
\end{tabular}

Notes: Events that occurred in $\geq 10 \%$ of total patients in the first year following VNS implantation are listed. Adverse events data were collected in Studies D-0I, D-02,

D-03, and D-2I. Second year adverse events data were not collected in Study D-2I. Year 2 patients $(N=344)$ include only patients reported to have data records in year 2 after completing year I. Adverse events have been coded using preferred terms based on COSTART. ${ }^{44}$

Abbreviation: VNS, vagus nerve stimulation.

\section{Publish your work in this journal}

Medical Devices: Evidence and Research is an international, peerreviewed, open access journal that focuses on the evidence, technology, research, and expert opinion supporting the use and application of medical devices in the diagnosis, treatment and management of clinical conditions and physiological processes. The identification of novel

\section{Dovepress}

devices and optimal use of existing devices which will lead to improved clinical outcomes and more effective patient management and safety is a key feature. The manuscript management system is completely online and includes a quick and fair peer-review system. Visit http://www. dovepress.com/testimonials.php to read real quotes from authors.

Submit your manuscript here: http://www.dovepress.com/medical-devices-evidence-and-research-journal 